Journal of

Women's Health and Gynecology

\title{
Large Prolapsing Uterine Fibroid and Severe Anemia in a Teenager: A Case Re- port
}

\section{Melanie Evans MD ${ }^{1, *}$, David Prokai $\mathrm{MD}^{2}$, Ellen E. Wilson $\mathrm{MD}^{3}$}

${ }^{1}$ Department of Obstetrics and Gynecology, UT Southwestern, Medical Center, Dallas, Texas

${ }^{2}$ Department of Reproductive Endocrinology, UT Southwestern Medical Center, Children's Health, Dallas, Texas

${ }^{3}$ Department of Obstetrics and Gynecology, Division of Pediatric and Adolescent Gynecology, UT Southwestern Medical

Center, Children's Health, Dallas, Texas

*Corresponding author: Melanie Evans, Department of Obstetrics and Gynecology, UT Southwestern Medical Center, Dallas, Texas, Tel: 713-775-5619, Email: melanie.evans@phhs.org

Received Date: October 06, 2019 Accepted Date: December 03, 2019 Published Date: December 05, 2019

Citation: Melanie Evans MD (2019) Large Prolapsing Uterine Fibroid and Severe Anemia in a Teenager: A Case Report. J Womens Health Gyn 6: 1-4.

\footnotetext{
Abstract

Background: Uterine fibroids are one of the most common tumors of the female reproductive tract, yet the prevalence is very low in patients under the age of 20 . Due to the rare incidence of fibroids in adolescence, the diagnosis may be delayed if imaging is not performed early in the workup for abnormal uterine bleeding (AUB).

Case: Here we present a case of a nulliparpous17-year-old Hispanic female with a 3-year history of AUB and anemia requiring a blood transfusion. After three years of medical management of her bleeding, the patient underwent imaging which revealed an $8 \mathrm{~cm}$ prolapsed intravaginal fibroid and a $5 \mathrm{~cm}$ intramural fibroid. She ultimately underwent fertility-sparing open myomectomy with a follow-up plan for serial ultrasounds every 6-12 months.

Summary and Conclusions: To our knowledge, this is the 16th case reported of uterine fibroids in females less than 18 years old. The management and treatment of uterine fibroids in the adolescent population should emphasize early imaging and preservation of fertility.

Keywords: Fibroids; Myoma; Leiomyoma; AUB; Anemia
} 
Introduction

Uterine fibroids (myomas, leiomyomas) are one of the most common tumors of the female genital tract. The incidence of fibroids increases with age and rises to over $40 \%$ in women over age 50. [1]. They are very rare in the adolescent population, with an incidence rate as low as $0.4 \%$ [2].

The most common cause of abnormal uterine bleeding in the adolescent population is chronic anovulation, either due to delayed maturation of hypothalamic-pituitary-ovarian axis, or an endocrine disorder such a polycystic ovary syndrome. Other causes include sexually transmitted infections, pregnancy, and bleeding disorders such as von Willebrand disease or idiopathic thrombocytopenia (ITP). As structural anomalies such as submucosal polyps/fibroids, or vagina/uterine cancers are extremely rare, imaging is often deferred until a workup for other more common causes are ruled out or medical treatment fails.

Here we present a case of a 17-year-old with abnormal uterine bleeding and severe anemia resulting from a large prolapsed cervical fibroid and a large intramural fibroid.

\section{Case Report}

Here we present a case of a nulliparous 17-year-old Hispanic female with a 3-year history of abnormal uterine bleeding and anemia, which had been treated unsuccessfully with oral contraceptive pills (OCPs). This was followed by tranexamic acid, IV Premarin, and ultimately leuprolide acetate over the span of 2.5 years. Multiple blood transfusions were also required during this time period. The patient was without other medical issues, but family history was significant for the maternal history of uterine fibroids requiring a hysterectomy. Due to the persistence of symptoms, a pelvic ultrasound followed by a pelvic magnetic resonance imaging (MRI), revealed a large intravaginal $8 \mathrm{~cm}$ prolapsed fibroid as well as a $5 \mathrm{~cm}$ intramural/submucosal fibroid. Office biopsy of the vaginal mass returned with benign pathology showing uterine fibroid.

The patient was then referred to our institution for fertility-preserving myomectomy. Physical exam revealed a 10 week size uterus that was mildly tender, and a large mass filling the vagina, clinically consistent with a prolapsed leiomyoma. Her presenting hemoglobin was $6.3 \mathrm{~g} / \mathrm{dL}$, and she was admitted the evening before surgery for transfusion of 2 units of packed red blood cells with an appropriate rise in her blood counts.

The patient underwent a laparotomy and myomectomy, removing a $5 \mathrm{~cm}$ intramural/partially submucosal fibroid as well as an $8 \mathrm{~cm}$ vaginal fibroid; the latter found to be originating from the upper cervical canal and removed via vaginal morcellation. The pathology report showed no necrosis, elevated mitotic activity, or nuclear atypia. The specimen did stain positive for desmin, caldesmeron, estrogen and progesterone receptors, and the final pathological diagnoses were a benign leiomyoma. The patient had an uncomplicated postoperative course with the cessation of her abnormal uterine bleeding as a result. Follow-up plans were to follow with serial ultrasound every 6-12 months for signs of recurrence. At a 1-year follow-up patient had no signs of recurrence of fibroid on ultrasound and her anemia had resolved. She did, however, have sonographic and clinical evidence of PCOS, and was restarted on OCPs.

\section{Discussion}

Few cases of uterine fibroids have been reported in patients under twenty years of age $[10,13]$. Most of these studies have dealt with patients that have had solitary symptomatic fibroids $[1,2,8,9,10,11,12,14]$, but others have involved multiple fibroids including one case with up to 19 separate fibroids [3].To our knowledge, this is the 16th reported case of uterine fibroids in a female less than 18 years of age, and the first reported case of an upper cervical fibroid that prolapsed to fill the vagina.

Multiple causes of uterine fibroids have been documented related to hormonal influence and genetics. Fibroids are estrogen-dependent and may enlarge during exogenous hormone use and pregnancy. Inheritance has also shown to play an important role in the pathogenesis of uterine fibroids and has been seen $2.2 x$ times more frequently among first degree relatives in families with two or more verified cases of fibroids [4]. Cytogenetic abnormalities involving chromosomes 6,7,12 and 14 have also been reported [5]. Our patient had reported a positive family history of fibroids in her mother.

Given that adolescents benefit from fertility-sparing surgery, a thorough preoperative workup using multiple imaging modalities may be necessary in order to make the correct diagnosis and plan the best operative path to take. Some patients with submucous myomas will benefit from a hysteroscopic approach alone, whereas intramural fibroids will need a laparoscopic or mini-laparotomy approach. As in adults, the initial work-up for a pelvic mass is sonography given its ease of availability and low cost. MRI imaging is considered the most accurate form of fibroid detection and has also been more useful in differentiating between other possible diagnoses including adenomyosis, adnexal tumors, and myometrial contractions [15]. MRI has a higher specificity, sensitivity, positive predictive value, and di- 
agnostic accuracy in detecting uterine sarcomas from fibroids, and these values increase when MRI is used in association with other lab tests for malignancy such a tumor marker, specifically lactate dehydrogenase [6]. MRI findings of mass degeneration, rapid mass growth, and free fluid in the pouch of Douglas have all been associated with leiomyosarcoma. Our patient had both pelvic ultrasound followed by MRI to better delineate mass characteristics.

While imaging can be a good initial diagnostic tool, true diagnosis cannot be made without pathologic evaluation. It can be particularly difficult to differentiate benign from malignant histology in adolescent patients as over $50 \%$ of recorded cases have shown malignant characteristics, but have final pathology consistent with benign myomas [3]. Unlike in adults, fibroids in adolescents can show increased cellularity, nuclear atypia, and increased mitotic figures [13,14]. Immunohistochemical markers such as desmin and h-caldesmon may help to differentiate benign from malignant pathology. Our patient's histology did not exhibit any malignant features but did stain positive for desmin and caldesmon.

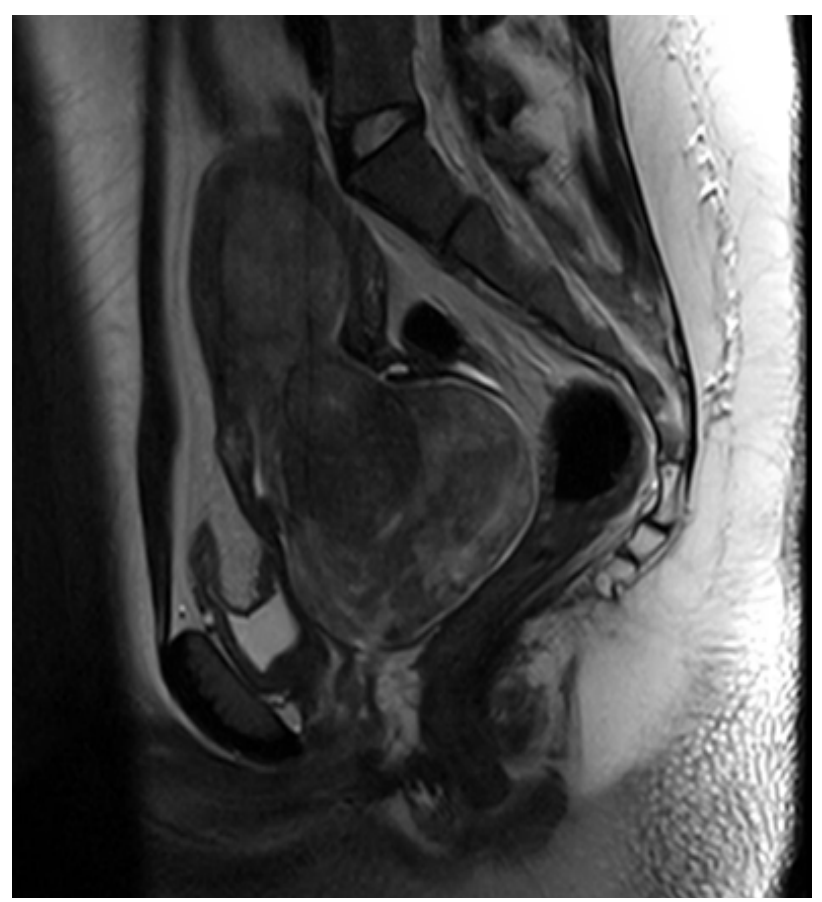

Figure 1
The management of fibroids in adolescents should be conservative. Asymptomatic small fibroids most often do not need surgical intervention whereas follow-up serial imaging is indicated. If surgery is indicated, myomectomy is the first choice given it is fertility-sparing. The use of preoperative gonadotropin-releasing hormones has been used in the adolescent population in an attempt to decrease the size of the fibroid and minimize intraoperative complications. However, in one study, the administration of leuprolide acetate to a 14-year-old patient was followed by an inflammatory reaction and increased symptoms that necessitated an earlier surgery [3]. Both open and laparoscopic approaches to surgery have been described in the adolescent population and based on patient factors and uterus size [9].In our patient, surgical management was necessary given the severity and duration of symptoms. An open surgical approach was chosen for fibroid size and location.

Fibroid recurrence has been variable among case studies but seems to be rarer in the adolescent population [1]. One study documented no recurrence at a 2-year follow-up [1], whereas a second study documented no recurrence at 5 years [11]. Another study showed a patient that had recurrence at both one and two year marks, requiring further surgical procedures [8]. Our patient showed no recurrence at a one-year follow-up.

While fibroids are rare in adolescents, one should have a high degree of suspicion if there is no response to symptoms with conservative measures. Imaging is an important diagnostic tool to help characterize fibroids in the adolescent population and should be done early in the work-up of abnormal bleeding. If surgical management is necessary, every effort should be made to preserve uterine integrity for future childbearing in this population. 


\section{References}

1) Maggiore, Umberto Leone Roberti, et al. (2013) A Case of Large Uterine Myoma in a 14-Year-Old Girl. Journal of Gynecologic Surgery, 29: 83-87.

2) Zimmermann, Anne, et al. (2012) Prevalence, Symptoms, and Management of Uterine Fibroids: An International InternetBased Survey of 21,746 Women. BMC Women's Health 1.

3) Tsili, Athina C, et al. (2010) Fibromatous Uterus in a 16-YearOld Girl: A Case Report. Case Reports in Medicine 1-5.

4) Vikhlyaeva E.m, et al. (1995) Familial Predisposition to Uterine Leiomyomas. International Journal of Gynecology \& Obstetrics 51: 127-131.

5) A. H. Ligon and C. C. Morton (2000) Genetics of uterine leiomyomata. Genes Chromosomes and Cancer, 28: 235-245.

6) E. Murase, E. S. Siegelman, E. K. Outwater, L. A. Perez- Jaffe,et al. (1999) Uterine leiomyomas: histopathologic features, MR imaging findings, differential diagnosis, and treatment. Radiographics 19: 1179-1197.

7) H. Hricak, D. Tscholakoff, and L. Heinrichs (1986) Uterine leiomyomass: correlation of MR, histopathologic findings, and symptomss. Radiology 158: 385-391.

8) Wright KN, Laufer MR (2011) Leiomyomas in adolescents. Fertil Stertil 95.

9) Augensen, K. Case Report (1981) Uterine myoma in a 15-yearold girl. ActaObsgtet Gynecol Sc and 60:591.

10) K. R. Fields and L. S. Neinstein (1997) Uterine myomas in adolescents: case reports and a review of the literature. Journal of Pediatric and Adolescent Gynecology 9: 195-198.

11) deRooy CGM, Wiegerinck MAHM (1986) A 15-year-old girl with an explansively growing tumor. Eur J Obstet Gynecol Reprod Biol 22: 373-377.

12) Heimer G et al: (1991) Uterine Myoma Causing Uremia in a 15-year-Old Girl. Gynecol Obstet Invest 32:247-248.
13) Morad NA et al. (1993) Cellular Uterine Myoma Causing Vaginal Bleeding in a 15-year-old girl. Aust NZ Obstet Gynaecol $33: 211-213$.

14) Diesen DI et al. (2008) Uterine Leiomyoma in a 14-Year-Old Girl. Eur J PediatrSurg 18: 53-55.

15) Perkins et al. (2009) Uterine Leiomyoma in an Adolescent Female. Journal of the National Medical Association. 101: 611613.

Submit your manuscript to a JScholar journal and benefit from:

ब Convenient online submission

I Rigorous peer review

- Immediate publication on acceptance

ब Open access: articles freely available online

9. High visibility within the field

- Better discount for your subsequent articles

Submit your manuscript at http://www.jscholaronline.org/submit-manuscript.php 